\title{
Modeling a UNI-Mosfet Switch for Single Phase System Application
}

\author{
Shashi kumar K., Venkataseshaiah C., K. S. Sim
}

\begin{abstract}
The concept of replacing mechanical switcher using Solid State Devices (SSR) as the medium of relaying high power AC load has already been recommended five decades ago using Dual Thyristor or Triac. The rapid growth in SSR field has now resulted in using dual IGBTs, dual JFETs, dual MOSFETs or dual HEMTs semiconductor devices to control high powered AC loads. One of the drawbacks of all the systems above is that they all using two semiconductor devices to operate for both half cycle of the single phase AC waveform, with either anti-parallel or anti series-arrangement incorporated. We have modeled a novel SSR, using only one MOSFET semiconductor device with a new SSR module packaging. This novel Uni-MOSFET SSR is capable of operating on both half cycles of an AC waveform and eliminating problems associated with dual semiconductor SSR system. Moreover, in this paper we have explained the simulations proving that the Uni-MOSFET SSR is able to control resistive load and able to compensate capacitive and inductive as a SVC, which is as good as its predecessor the Dual-Thyristor SSR. The draw back would be the complexity of the control signals of the Uni-MOSFET SSR for TCR but we have managed to simplify using equations. Our novel SSR is cost efficient, low noise and works well as a load switcher, circuit breaker or compensation switch for single phase electrical network system.
\end{abstract}

Index Terms: SSR, MOSFET, SVC, PFC, TCR, TSC.

\section{INTRODUCTION}

Mechanical switcher or relays were the popular devices for a long time. They are used as AC load switches or selector, circuit breakers or trippers and compensation selector. In our previous paper [1] and [2] we discussed how mechanical relays were used as compensation selectors. However, the need for compact smaller and durable switches gave birth to Solid State Relays fifty years ago. The Dual-Thyristor SSR topology for AC power system remains the same during these decades [3]. Although new topology with newer semiconductor types used the Super Junction MOSFET, the topology still needed dual semiconductors to control each half cycle of the AC waveform separately [4]. There are many disadvantages associated with pre-successor topology, for example the loud switching noises will cause un-similar dual control signals.

Our proposal is to use our new Uni-MOSFET topology incorporated as a novel SSR module packaging. In our

Revised Manuscript Received on August 19, 2019.

Shashikumar K., PhD Researcher at FOE, Multimedia University, Cyberjaya, Selangor, Malaysia. University, Bukit Katil, Melaka, Malaysia.

K. S. Sim, PhD Research Co-Supervisor at FET, Multimedia University, Bukit Katil, Melaka, Malaysia.
Venkataseshaiah C., PhD Research Supervisor at FET, Multimedia

previous paper [5] we have proven how the Uni-MOSFET topology works on capacitive load for TSC compensation. In

this paper we are intend to prove that Uni-MOSFET could also be used in inductive load for TCR compensation with detailed illustration using MATLAB Simulink simulator for capacitive, inductive and resistive loads. Our novel Uni-MOSFET SSR module can be used as load switching, circuit breakers or compensation switching. The advantage of our novel module is cost reduction and THD reduction due to the use of only one control signal line. Authors of paper [6], [7] and [8] showed simulation of FC-TCR compensation in single phase AC system using Dual-Thyristor for TCR branch. They have elaborated in detail, the variation of the inductor current waveforms and THD noise in these papers. Dual-Thyristor SSR can be used for different purposes like controlling and compensation as shown in Figure 1. Voltage Transformer and Current Transformer are used to measure the P, V, I, PF and zero-crossing signal generation. These signals are processed by AtMega328 micro-controller as illustrated in our paper [1] and [2]. To control the capacitor and inductor we use SSR Crydom D24-50-D-H-10 with built in random turn ON opto-coupler MOC3020 (Figure 2 (a)) that can take up to 50A inrush current. For turning ON/OFF the resistive load, we use the SSR Crydom D24-50-D-H with built in zero-crossing turn ON opto-coupler MOC3041 and it also could take up to 50A inrush current too. For the resistive load turn ON, a simple high and low signal given to the SSR and the internal zero-crossing circuit of the SSR will ON with a phase delay of 3 degrees. The SSR Crydom D24-50-D-H only can work with minimum power factor difference of 0.5 degrees. Furthermore, Crydom SSR has two gate signal pins for controlling each SSR Thyristor individually.

Figure 3 shows MATLAB simulink model for the SSR Cyrdom D24-50-D-H-10 we have tested. The model could be loaded with capacitive, inductive or resistive load.

The best pulse width for triggering the SSR Thyristors is $1 \%$ which equals to 3.6 degrees. For both capacitive and inductive load, the SSR Thyristor gate 1 signal phase is delayed from 0 to 180 degrees with a fixed $1 \%$ pulse width as shown in Figure 4 (b) and Figure 5 (b). SSR Thyristor gate 2 signals delayed 180 degrees with respect to gate signal 1 for the negative source voltage cycle quadrant shown in Figure 4 (c) and 5 (c).

\section{Published By:}




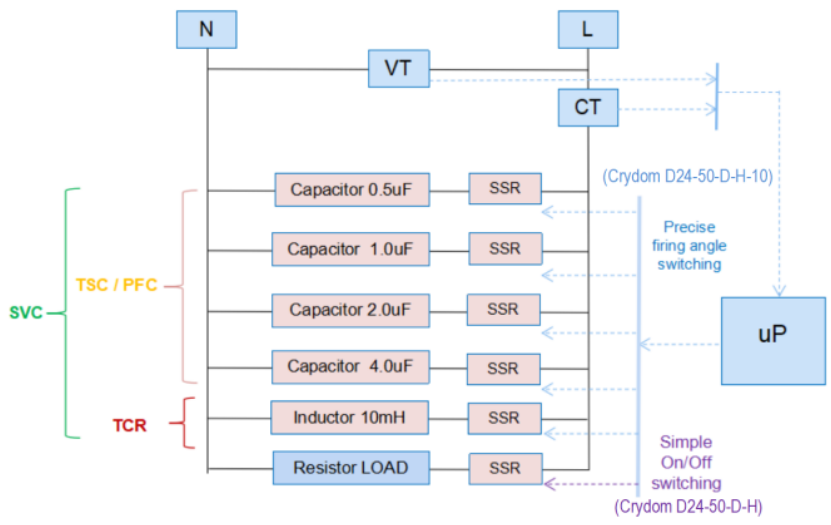

Figure 1: The Dual-Thyristor SSR configuration used for load and compensation control.
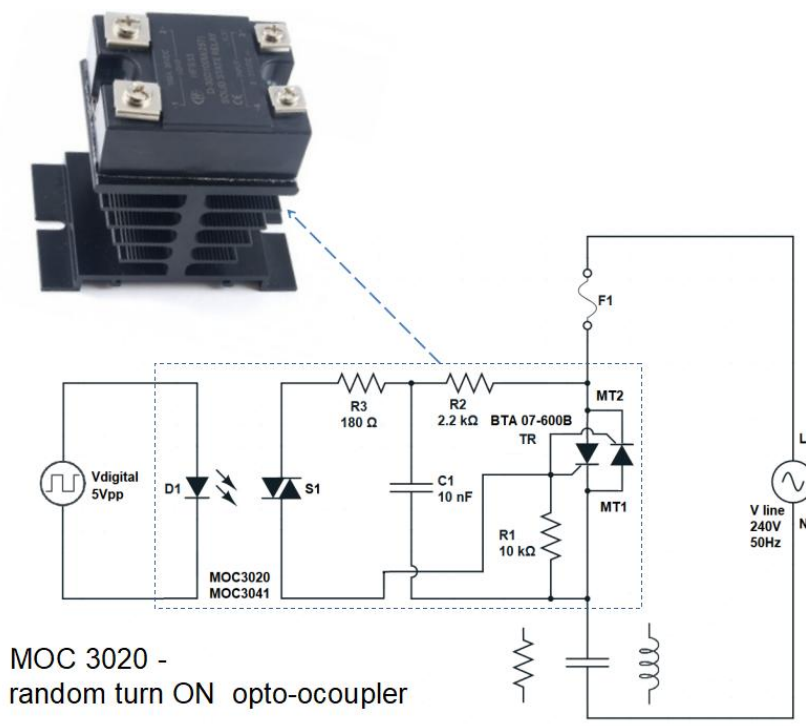

(a)

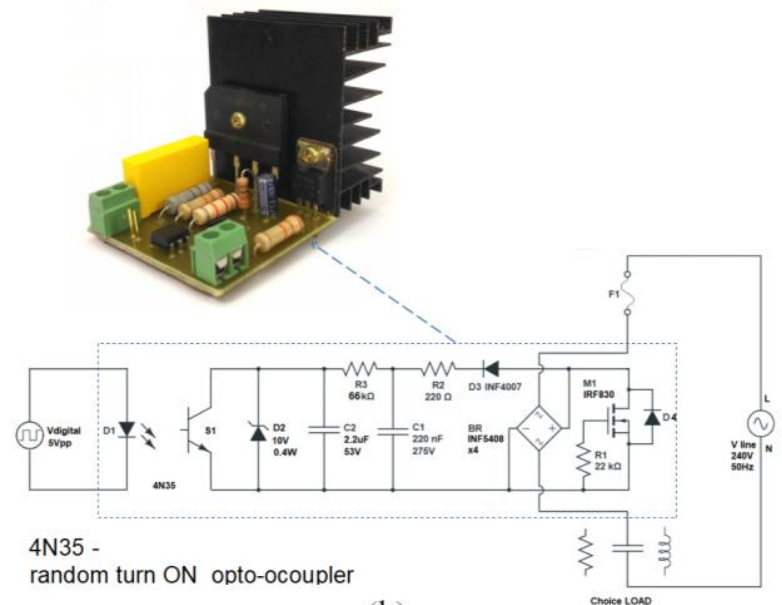

(b)

Figure 2: (a) SSR circuit build using a TRIAC with choice of opto-couple configuration of random turn $\mathrm{ON}$ or zero-crossing turn ON, whereas (b) Uni-MOSFET SSR circuit prototype.

\section{DUAL-ThYRISTOR SSR SimUlation RESUltS}

From the Dual-Thryristor simulation experiment in Figure 3 , we can the conclude that the effect of compensation for capacitive TSC best happens when the capacitor voltage charged the maximum peek as highlighted in red box in Figure 4 (f) between 70 degrees - 110 degrees. As the standard practice, a fixed 90 degrees applied. This would turn on the capacitor for PFC, keeping in mind that the capacitor in TSC could not be varied in value using this method. Also, as highlighted in the green box in Figure 4 (g) and $(\mathrm{h})$, at this point the highest power factor and power is absorbed by the capacitor.

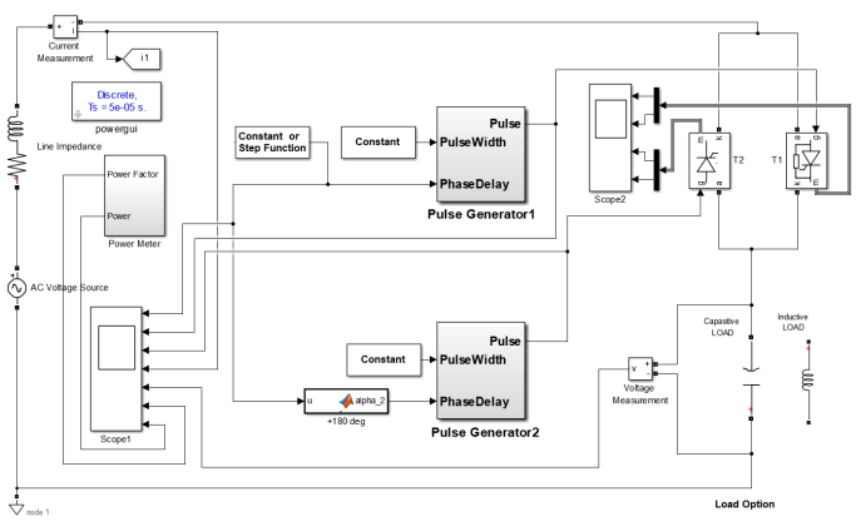

Figure 3: Dual-Thyristor SSR MATLAB Simulink model for capacitive and inductive compensation.

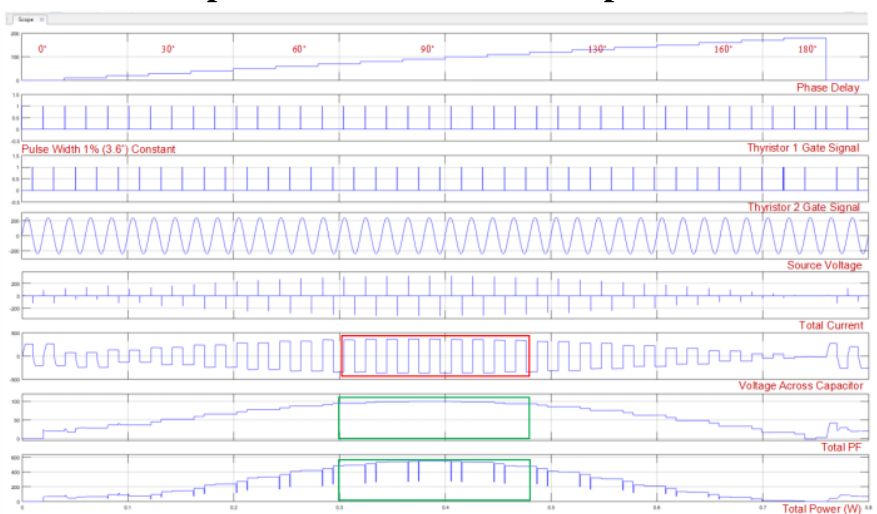

Figure 4: Waveform generated at the Scope 1 of Figure 3 when TSC compensation applied. The marker red and green boxes are useful conduction angels

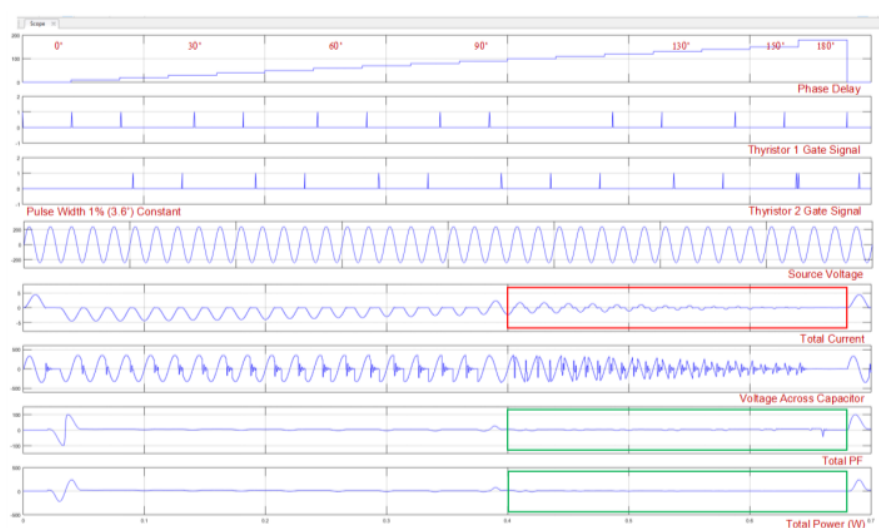

Figure 5: Waveform generated at the Scope 1 of Figure 3 when TCR compensation applied. The marker red and green boxes are useful conduction angels.

We are highlighting this for us to demonstrate and prove that our Uni-MOSFET module later in this paper will also give similar results. Similarly, we have

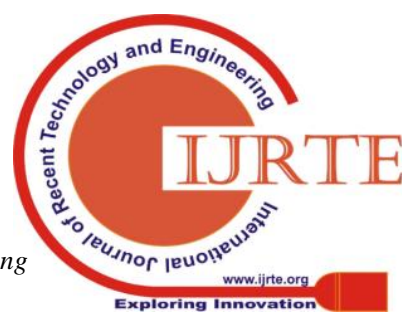


highlighted in the red box in Figure 5 (e) to show that for the inductive TCR compensation to take effect with variable PFC or SVC, the best triggering phase angle used is from 100 degrees to 180 degrees for Thyristor gate 1 and Thyristor gate 2 signals is 180 degrees apart throughout. At this phase, the inductor current is perfectly 90 degrees apart from source voltage waveform, giving results of zero power absorb and zero

power factor as per the equation $\cos \theta$ in theory. Near zero power and zero power factor could be seen in our simulation in Figure $5(\mathrm{~g})$ and $(\mathrm{h})$ and is highlighted in green box. At this point variation of inductor current from 180 degrees to 100 degrees will affect the fix parallel capacitor to differ to lower values. The full parameters were fully tested and explained in our previous paper [5].

\section{UNI-MOSFET SSR SIMULATION RESULTS}

The purpose of elaborating the above simulation for Dual Thyristor SSR is to prove that our Uni-MOSFET SSR meets the current practice of SVC standards when implemented as compensator. From the figure above, we can now demonstrate how we attempted to obtain similar waveform results for our novel Uni-MOSFET SSR model. Our new Uni-MOSFET SSR MATLAB simulation model as shown in Figure 6 consists of only one semiconductor switching device and four bridged connected diodes. Only two diodes at a time are allowed to work in the forward direction in a half cycle of $50 \mathrm{~Hz}$ supply and the MOSFET works on both half cycle in the same direction. We next applied capacitive load to the simulation model Figure 6 and we ingeniously managed to identify the target area by fixing the duty cycle to a random value of 50 degrees and volume down the pulse width from $100 \%$ to $0 \%$ as shown in Figure 8 (a) and (b). As anticipated we have manage to spot the targeted area and highlighted in red and green box in Figure 8 (c) and (d).

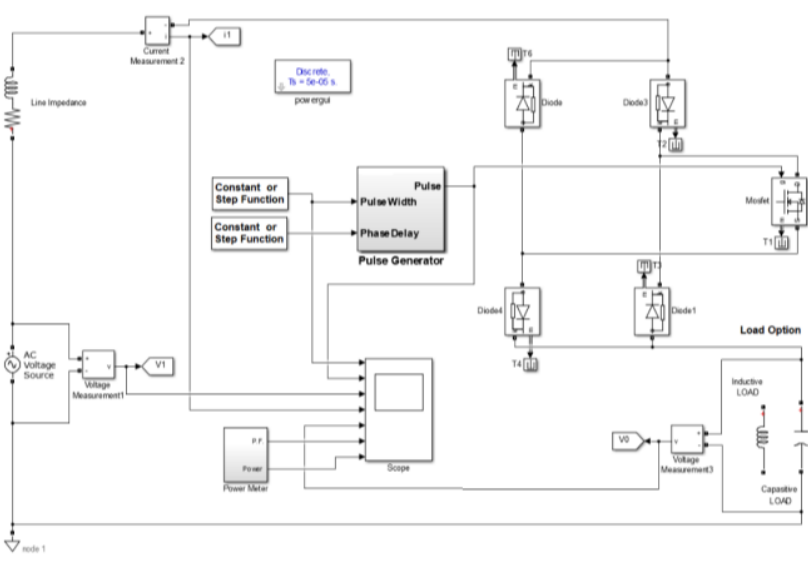

\section{Figure 6: Uni-MOSFET SSR MATLAB Simulink model for TSC and TCR compensation.}

Both the highlighted box's area are similar and match with Figure 4 (f) and (h) of the Dual-Thyrsitor SSR TSC compensator at $10 \%$ pulse width setting in our Uni-MOSFET SSR TSC. With this we conclude a $10 \%$ pulse width setting is ideal for Uni-MOSFET SSR TSC rather than the $1 \%$ pulse width we used in Dual-Thristory SSR TSC model. However, we noted that the waveform has similarity but no consistence.
Further analysis resulted in better results. Figure 9 (b) shows with a fixed $10 \%$ pulse width setting and changing the size of phase delay from 0 degrees to 180 degrees a beautiful butterfly waveform emerges as shown in Figure 9 (e). Now we can confidently say we that we have successfully designed this Uni-MOSFET SSR TSC model to work for capacitive compensation at the following triggering phase angle of 30 to 40 degrees or optionally at another phase angle of 130 to 140 degrees. At these two points, the relevant green box's in Figure 9 (f) and (g) is concurrent with standard TSC results in Figure $4(\mathrm{~g})$ and $(\mathrm{h})$ too. We feel the results are selfexplanatory and fulfill the requirement of a new topology SVC. Next we applied inductive load to the simulation model in Figure 6. After many simulations, we finally broke the barrier and for the first time we could see the depth of the Uni-MOSFET SSR TCR simulation results in Figure 10 and Figure 11. Figure 10 (b) shows a random and reasonable value of $70 \%$ fixed pulse width applied while the phase shift knob increased from 0 to 180 degrees. Amazingly we observed two sweet spots similar to our Uni-MOSFET SSR TSC simulation in Figure 8. This shows that we have again two options to play around with for our Uni-MOSFET SSR TCR. The two sweet spots highlighted in red box in Figure 10 (c) resembles to Dual-Thryistor SSR TCR in Figure 5 (e). Furthermore, the power factor and power is very low (nearly zero at this two sweet spots) as highlighted in the green boxes in Fig. 10 (e) and (f) also resembles TCR standards as shown in Fig.5 (g) and (h).

In power electronics, a rule states that a current driven device like the inductor and a voltage driven device like the MOSFET will not work along best but we have proven otherwise. Here in our simulation as seen in Figure 10 (d) (sweet spots), there are no large spikes but outside the spots large spike occur due to invalid triggering values applied. We can now conclude that the two best values suited setting for our Uni-MOSFET SSR TCR model is at pulse width $70 \%$ and phase delay 60 and 150 degrees. As we need to adjust the volume of inductor current to suit the purpose of the variable PFC, we again need more derivation of formula to achieve TCR standard as cascading down current waveform as shown in Figure 5 (e). To achieve this we managed to devise a revolutionary novel idea for the pulse generator control unit. We have taken one of the sweet spots in Figure 10 at phase delay 60 degrees and simulation a final solution for the pulse generator controller using equation 1 . The equation satisfies our TCR standards pairing with Uni-MOSFET SSR TCR. Equation 1 curve in Figure 7 (a) is a correlation equation between two linearly exponential curve of Uni-MOSFET SSR TCR pulse delay and phase width from Figure 7 (b). Equation 2 is also a correlation equation between two linearly exponential curve of Dual-Thyristor SSR TCR phase delay and Uni-MOSFET SSR TCR pulse width from Figure 7 (b).

Pulse Delay $=-0.5($ Phase Width $)+95$

Thyristor $_{(\text {Pulse Delay })}=-\left(\right.$ MOSFET $_{(\text {Phase Width })}+190$

Now we could vary the inductor current of the TCR using these equations and 
if-else statement as the control unit for the pulse generator as shown in Figure 12. Our control unit design in Figure 12 correlates with the curve in Figure 7. This indicates the extent to which one variable increases as the other decreases as seen in Figure 7 (b) blue and orange coloured lines. In Figure 7 (b) the TCR control signal curve at the left tip is exponential landing at $5 \%$ pulse width and 90 degrees phase delay, where these signals are used to gradually halt the inductor compensation current to zero in our Uni-MOSFET SSR TCR model.

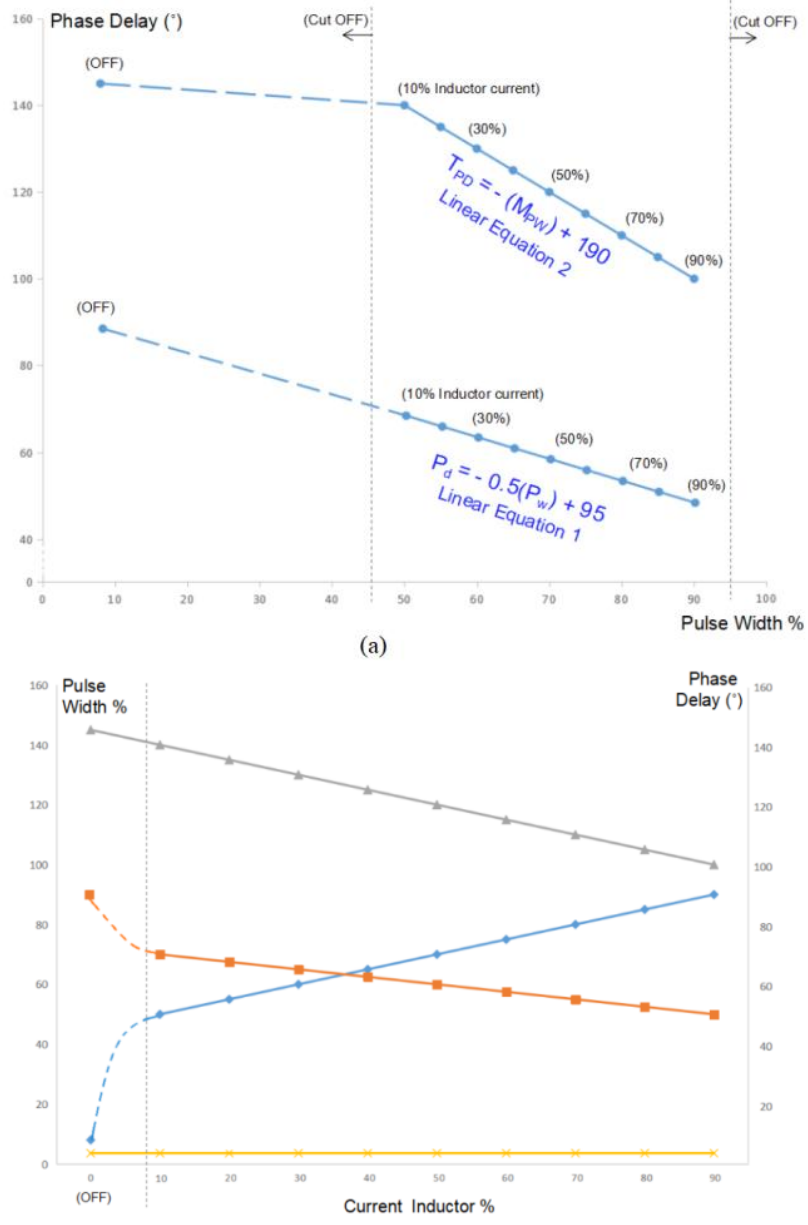

(b)

Figure 7 (a) shows two correlation line between two sets of lines from (b). And (b) shows variation for TCR pulse generator input control parameters for Dual-Thyristor and Uni-MOSFET.

MATLAB simulink model in Figure 12 used to easy controlling the pulse generator by adjusting a single input value from $50 \%$ to $90 \%$ as related to pulse width and percentage of current increment in the inductor from $10 \%$ to $90 \%$ and the model automatically adjusts the phase delay accordingly. If the input adjusted value exceeded the range of $50 \%$ to $90 \%$ then the model in Figure 12 will automatically reduce the inductor current to zero with fixing the pulse generator parameters to $5 \%$ and 90 degrees. The detail computation for gate signals to generate pulse parameters for Dual-Thrysitors TCR was shown in our previous paper [5]. This paper illustrated how the solutions from the
Dual-Thrysitors TCR gate signals (140 - 100 degrees) are modeled to calculate and generate new pulse parameters for our novel Uni-MOSFET SSR TCR using the model from Figure 12. In other words, we have simplified the complicated control signal with the need to control one parameter only and the model does the rest of the computation with ease using both equations.

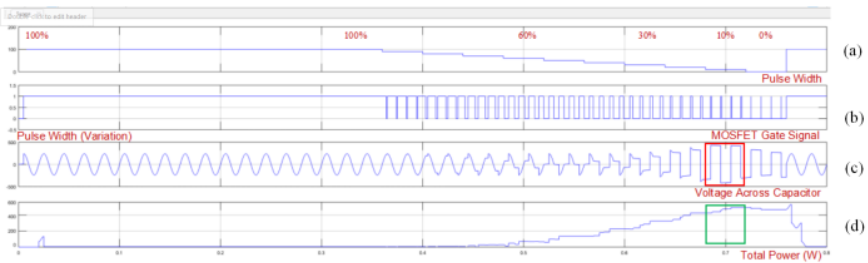

Figure 8 shows Uni-MOSFET capacitive TSC first part simulation solutions.

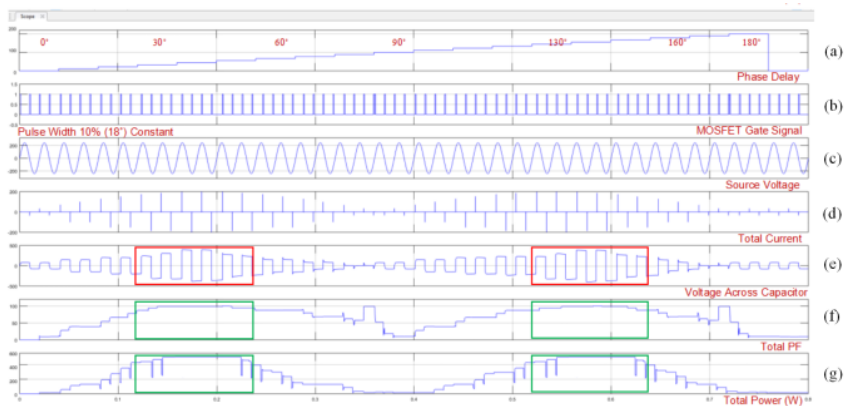

Figure 9 shows Uni-MOSFET capacitive TSC second and final simulation results.

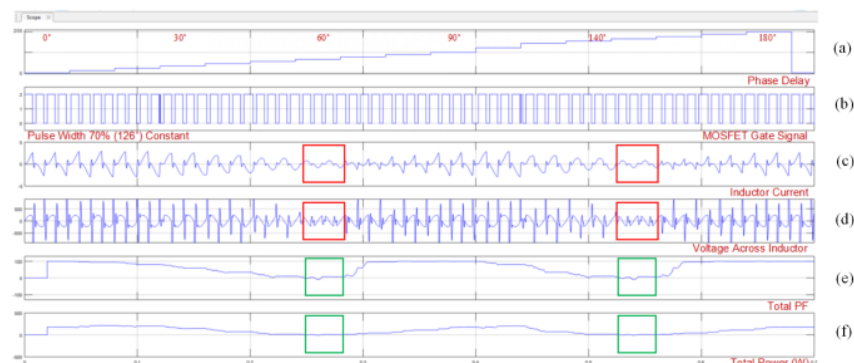

Figure 10 shows Uni-MOSFET inductive TCR first part simulation solutions.

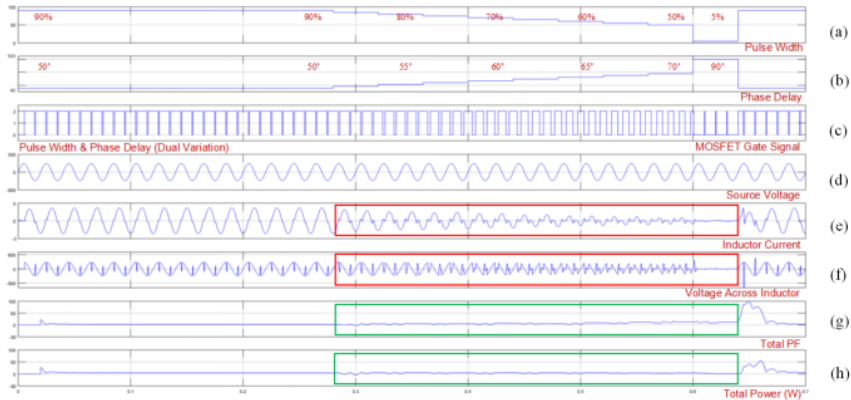

Figure 11 shows Uni-MOSFET inductive TCR second and final simulation results

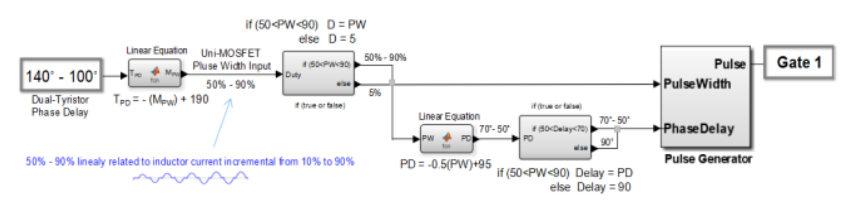

Figure 12 shows control unit designed for

Published By:

Blue Eyes Intelligence Engineering

\& Sciences Publication 
Uni-MOSFET inductive TCR compensation.

The full extent of the simulation of our novel Uni-MOSFET SSR TCR is shown in Figure 11, where the pulse width decrements inputted by user as shown in Figure 11 (a) generates automatic incremental parameters for phase delay in Figure 11 (b). Figure 11 (e) shows the current in the inductor 90 degrees differs to source voltage in Figure 11 (d) and decrements to zero as the used exceeded the range permitted. Comparing the standard practice waveforms from Dual-Thyristor SSR TCR waveform in Figure 5 (e), (f), (g) and (h) to our Uni-MOSFET SSR TCR waveform in Figure 11 (e), (f), (g) and (h), they match exactly for inductor current, inductor voltage, power factor and power of the system. Furthermore, our system do not strain the MOSFET as there are no large spikes generated during switching of the inductor as observed in the Figure 11 (f). The inductor voltage in Figure 11 (f) has no spikes compared to Figure 10 (d) where there is imperfection switching at outside of the sweet spots.

Therefore, we could conclude that the Uni-MOSFET SSR has a drawback as it needs complicated pulse generator controller but works better than Dual-Thrysistor SSR because it delivers less THD noise as a single switching device. Table 13 shows the summary of our findings for pulse generator control signal. Some of the findings tabled we have not discussed or elaborated in this paper as the procedures are the same. Uni-MOSFET SSR has options of trigger range at different quadrant of the full wave cycle for both TCR and TSC. The best quadrant for SVC combination the TCR quadrant 2 and TSC quadrant 1 where it follows Dual-Thyristor control method as show in Table 13 TCR quadrant 2 and TSC quadrant 1. Quadrant 1 works on triggering angle 0 to 90 degrees and 180 to 270 degrees wheres Quadrant 2 works on triggering angle 90 to 180 degrees and 270 to 360 degrees. For Uni-MOSFET SSR load controller module any quadrant would work perfectly for capacitive or inductive loads.

Table 13 shows summary of pulse generator control signal parameters respect to different loads.

\begin{tabular}{|c|c|c|c|c|c|c|c|}
\hline $\begin{array}{c}\text { LOAD } \\
\text { Type }\end{array}$ & $\begin{array}{c}\text { SSR } \\
\text { Switching } \\
\text { Type }\end{array}$ & $\begin{array}{c}\text { Pulse } \\
\text { Width } \\
\text { gate1 }\end{array}$ & $\begin{array}{c}\text { Pulse } \\
\text { Width } \\
\text { gate2 }\end{array}$ & $\begin{array}{c}\text { Phase } \\
\text { Delay } \\
\text { gate1 }\end{array}$ & $\begin{array}{c}\text { Phase } \\
\text { Delay } \\
\text { gate2 }\end{array}$ & $\begin{array}{c}\text { Control } \\
\text { Mode }\end{array}$ & $\begin{array}{c}\text { Trigger } \\
\text { Quadra } \\
\text { nt }\end{array}$ \\
\hline Capasitive & Dual-Thyristor & $1 \%$ & $1 \%$ & $70^{\circ}-110^{\circ}$ & $250^{\circ}-290^{\circ}$ & Fix $90^{\circ} \& 270^{\circ}$ & 1 \\
\hline Inductive & Dual-Thyristor & $1 \%$ & $1 \%$ & $100^{\circ}-180^{\circ}$ & $280^{\circ}-360^{\circ}$ & Single Variation & 2 \\
\hline Resistive & Dual-Thyristor & $1 \%$ & $1 \%$ & $10.8^{\circ}$ & $190.8^{\circ}$ & Fix & 1 \\
\hline Capasitive & Uni-MOSFET & $10 \%$ & Nil & $30^{\circ}-40^{\circ}$ & Nil & Fix $35^{\circ}$ & 1 \\
\hline Capasitive & Uni-MOSFET & $10 \%$ & Nil & $130^{\circ}-140^{\circ}$ & Nil & Fix $135^{\circ}$ & 2 \\
\hline Inductive & Uni-MOSFET & $50^{\circ}-90^{\circ}$ & Nil & $70^{\circ}-50^{\circ}$ & Nil & Dual Variation & 1 \\
\hline Inductive & Uni-MOSFET & $140^{\circ}-180^{\circ}$ & Nil & $160^{\circ}-140^{\circ}$ & Nil & Dual Variation & 2 \\
\hline Resistive & Uni-MOSFET & $95^{\circ}$ & Nil & $8^{\circ}-10^{\circ}$ & Nil & Single Variation & 1 \\
\hline
\end{tabular}

\section{Conclusion}

We have shown a detail working simulation results for the conventional Dual-Thyristor SSR for varies loads. The Dual-Thyristor SSR simulation was usefull for us to design and compare results to our novel Uni-MOSFET SSR prototype model. Even though some control signals are complex for our novel model, we have simplified it with equations and tested with programmed hardware using AtMega328 micro-controller. Furthermore in this paper we have backed our finding with solid simulations results. The plus point of our system is that it has dual options of trigger control at different quadrant cycle, giving advantages for PPL and THD adjustments. Our new SSR module is much cheaper to manufacture because it uses only one switching semiconductor device, eliminating the need for an additional anti-parallel circuit and also reduces THD noise easily to $50 \%$. We have tested our system as a load switching, circuit breaker and compensation switching on single phase electrical system. We did not attempt on three phase system due to cost constraints. Our proposal for the future would be to design and implement the system for three phase. Another recommendation would be to attempt to use Uni-Thyristor modeling.

\section{REFERENCES}

1. Shashikumar K. et al. Juggling an Arduino for Multi-Meter, Load Profiling and Novel Waveform Capture Logger Application, 12 th International Colloquium on Signal Processing \& its Applications of IEEE, (2016) 119-123.

2. Shashikumar K. et al. Automatic Sequential Reactive Power Compensation and Harmonic Suppression at Loads using Appliance Clustering and Power Quality Monitoring, International Conference on Robotics Automation and Sciences (ICORAS) of IEEE, (2016) 1-6.

3. George Osborn, Solid State Catches Up in Relay Tace, Published in Electronic and Power IEEE, Volume 18, No. 8.9 (1972) 315-318.

4. M. Fernandez et al. Analysis of Solid State Relay Solutions Based on Different Semiconductor Technologies, Power Electronics and Applications IEEE (EPE'17 ECCE), (2017) 1-9.

5. Shashikumar K. et al. Fuzzified Single Phase Automatic Sequential Reactive Power Compensation with Minimized Switches, Journal of Telkomnika, 16(2): 889-899, (2018).

6. T. Vijayakumar et al. Harmonics Analysis of Thyristor Controlled Reactor Circuits, International Journal Computer and Electrical Engineering, Vol. 2, No. 1 (2010) 190-192.

7. Ali Kose et al. Modelling and Simulation of a Static VAR Compensator Based on FC-TCR, International Conference Renewable Energy Research and Application (2016) 924-927.

8. Dang Van Huyen et al. Design of Dynamic-Static Var Compensation based on Microcontroller for Improving Power Factor, International Conference System Science and Engineering (ICSSE) (2017) 186-190.

\section{AUTHORS PROFILE}

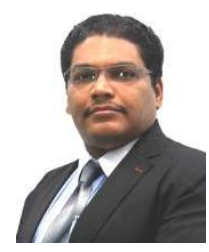

Shashikumar. $\mathrm{K}$ is with the Multimedia University, Cyberjaya, 75450 Malaysia. He is now a lecturer with Faculty of Engineering with a teaching experience of 15 years. He obtained his Master Degree in Power Electronics from University Putra Malaysia and final stages of his $\mathrm{PhD}$ as he is awaiting for his ViVa. He has industrial working experience and obtained certificate as a professional engineer from Board of Engineers Malaysia. He also published varies papers in the field of measurements and electrical compensators. (e-mail: shashikumar@mmu.edu.my). 
Modeling A Uni-Mosfet Switch for Single Phase System Applications

Venkataseshaiah C. is with the Multimedia University, Melaka, 75450 Malaysia. He is now an Associate Professor with Faculty of Engineering and Technology with teaching experience of 47 years. He obtained his $\mathrm{PhD}$ in year 1976 in the field of Electrical from Indian Institute of Technology Madras. He have published around 100 papers in the field of electrical engineering. He is also a life member of Indian Society for Technical Education. (e-mail: venkataseshaiah@mmu.edu.my).

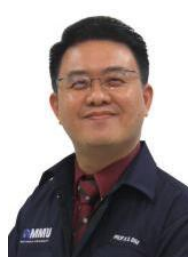

K.S. Sim is with the Multimedia University, Melaka, 75450 Malaysia. He is now a Professor with Faculty of Engineering and Technology and teaching for at least 20 years. He also has 15 years industrial working experience and obtained certificate as a professional engineer from Board of Engineers Malaysia. He obtained his $\mathrm{PhD}$ in Multimedia University and Masters of Engineering form National University of Singapore. He has various medals and top research scientists award in Malaysia. He have published 279 papers up to date. (e-mail: kssim@mmu.edu.my). 